\title{
Bronchiectasis in a positive HIV child: a case report
}

\author{
Shrestha L, Basnet S, Kanskar P, \\ Department of Pediatrics, Institute of Medicine, Kathmandu, Nepal
}

Correspondence Address: Dr. Laxman Shrestha, Department of Pediatrics, Institute of Medicine, Kathmandu, Nepal

Email:laxmanshree@yahoo.com

\begin{abstract}
Human immunodeficiency virus (HIV) infection has a wide spectrum of pulmonary disease which usually complicates the clinical course. Non- cystic fibrosis bronchiectasis is more common in children with HIV infection than in general population. Bronchiectasis in children with HIV if not diagnosed early and managed aggressively can lead to irreversible destruction of bronchial wall and surrounding tissue leading to significant morbidity. This might decrease the benefit of the antiretroviral therapy (ARV) treatment on the long run.
\end{abstract}

\section{Introduction}

The AIDS epidemic is arguably the most important global health problem of the last two decades. Previously known to be a uniformly fatal disease is now considered as a chronic and controllable disorder due to combination antiretroviral therapy and restoration of immune disease But, these treatments are expensive and difficult to distribute to the millions in need them. ${ }^{1}$

Human immunodeficiency virus (HIV) infection has been associated with a wide spectrum of pulmonary disease especially presenting in the first decade of life. ${ }^{2}$ The risk for the development of pulmonary disorders is strongly influenced by the severity of immunosuppression, use of intravenous injecting drugs, patients belonging to minority communities. The risk decreases with the use of ART and prophylaxis against common HIV- associated infections.1 These pulmonary diseases usually complicate the clinical course of the HIV infection. ${ }^{3,4}$

Bronchiectasis is an abnormal and permanent dilatation of the bronchi, most commonly the result of a previous infectious process triggering an ongoing inflammatory response leading to the damage of the bronchial wall beyond repair. ${ }^{5}$ The pathogenesis comprises infective, inflammatory and enzymatic elements. These interact to perpetuate continued airway damage in bronchiectasis leading to progressive airway and lung damages. The weakened bronchial wall is subjected to an abnormal pulling force from surrounding atelectatic lung, perhaps augmented by trapped secretions, leading to permanent dilatation. ${ }^{6,7}$

Reported incidence of non- cystic fibrosis bronchiectasis was about $1 \%$ in 19948 while in 2004 was $9.6 \% .{ }^{9}$ Out of the very few studies reporting cases of bronchiectasis in $\mathrm{HIV}$ infected individuals, the prevalence ranged from 15 to $42 \% .{ }^{10-16}$ Following the first case report from Oxford in $1992,{ }^{10}$ there are very few cases of HIV reported to have bronchiectasis although the prevalence of bronchiectasis is higher in HIV infected population. This might be because of the low index of suspicion and because of the fact that the easily available investigations like chest radiographs in the early stages may be normal or nonspecific.

Here we are reporting the case of bronchiectasis in HIV infected 7 years old child.

\section{Case summary}

A 7 years old, male child diagnosed as HIV positive 6 months back presented with 15 days history of dry cough associated with fever for 7 days. There was no history of chest pain, breathlessness, cyanosis and hemoptysis. There was no history of decrease in appetite or significant weight loss in the past 15 days.

The child was born as $3 \mathrm{~kg}$, term baby by spontaneous vaginal delivery at hospital. The boy studies in upper kindergarten and has an average performance. He was breast fed exclusively for 6 months followed by introduction of complementary food. At present he takes 2 major meals and 4 minor meals with adequate calorie intake. He has been immunized according to the National Program of Immunization. 
The child was investigated for failure to thrive at 2 years of age and diagnosed with pulmonary tuberculosis and treated with antitubercular drugs. There was past history of repeated episodes of fever and cough needing admissions in hospital and treated as pneumonia with antibiotics. There was history of recurrent ear discharge in the past.

Both the parents are HIV infected and a younger sibling has not been tested for HIV. The father was diagnosed as HIV positive 4 years back and mother was diagnosed 6 months ago. Both parents are not under antiretroviral therapy. There is no history of tuberculosis in the family.

On examination the child was thin built. The child was undernourished, stunted and wasted. There was generalized lymphadenopathy and clubbing. The child was in respiratory distress with oxygen saturation of $85 \%$ without oxygen. There were bilateral diffuse, coarse crepitations. Per abdominal examination revealed hepatosplenomegaly.

The child was admitted with the provisional diagnosis of pneumonia with distress in a child with HIV infection. Investigations revealed white blood cell count of 4180 cells $/ \mathrm{mm}^{3}$ with $42 \%$ neutrophils, $47 \%$ lymphocytes, $10 \%$ monocytes and $1 \%$ eosinophils. Hemoglobin was $10.3 \mathrm{gm} \%$ and platelet count was 106,000 cells $/ \mathrm{mm}^{3}$. The erythrocyte sedimentation rate was 57 in the 1 st hour. CD4+T cell count was 511 cells/uL and constituted $13 \%$ of the lymphocytes. There was no CD4+T cell count done previously. Three samples of gastric aspirate for tuberculosis was negative, gram stain showed gram positive cocci in pairs and chain but culture was negative. Mantoux test was negative. Chest radiography was suggestive of bilateral bronchiectatic changes and high resolution CT scan confirmed bilateral bronchiectasis.

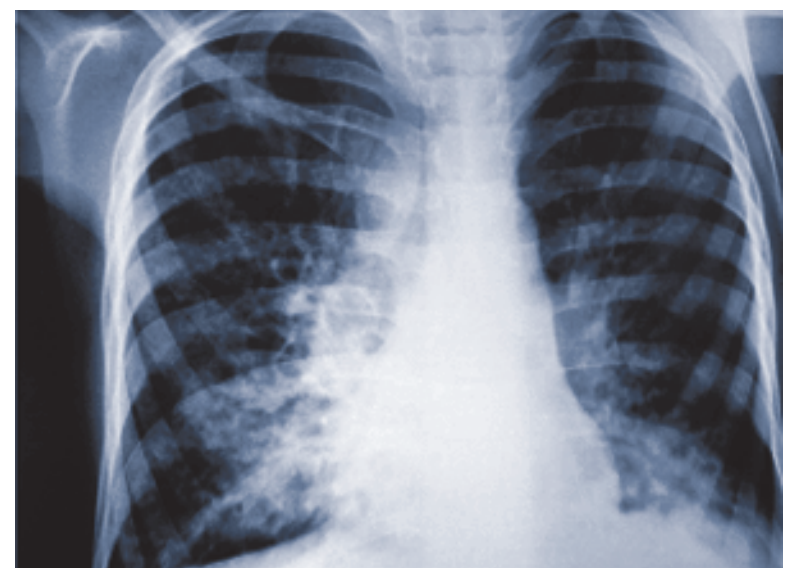

Chest X-ray showing bilateral bronchiectatic changes in both lower lung fields.

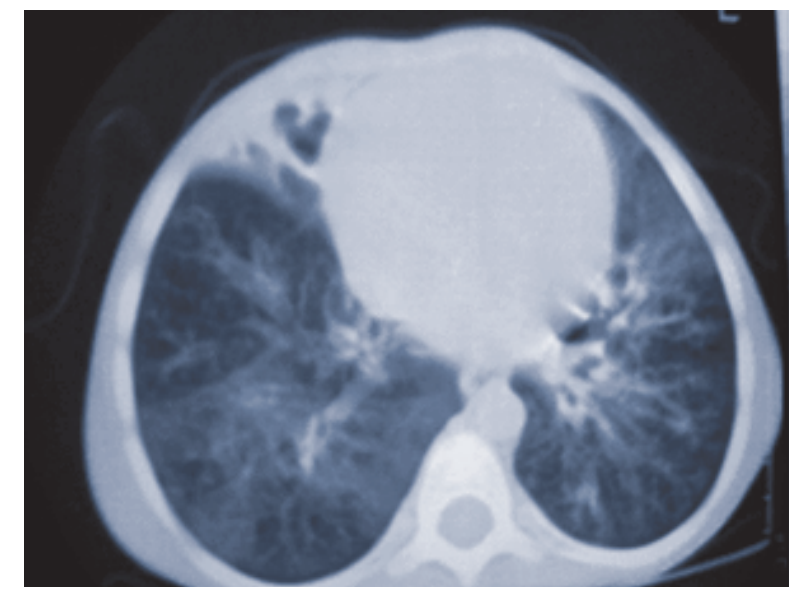

CT chest showing bronchietatic changes in right

upper, middle lobes and basal segment of left lower lobe.

With the diagnosis of bronchiectasis with secondary infection, he was treated with parental antibiotics, oral multivitamins and iron. Chest physiotherapy was done regularly. Cardiothoracovascular surgery consultation was done who suggested continuing the medical management as the disease involved both the lungs.

The child was discharged on multivitamins, iron, cotrimoxazole and azithromycin prophylaxis.

\section{Discussion}

Although the prevalence of bronchiectasis in children with $\mathrm{HIV}$ infection is quite higher than in non-HIV infected cases, bronchiectasis is rarely discovered in children with HIV infection because of the low index of suspicion. Bronchiectasis should be suspected in children who present with history of persistent or intermittent cough with or without purulent sputum and inspiratory crackles on physical examination. Other clinical findings suggesting bronchiectasis includes features like halitosis and clubbing. Our case presented with persistent cough without sputum.

Although bronchiectasis is defined by histhopathological examination of lung biopsies, a diagnosis can be inferred based on chest X- ray, pulmonary function tests and high resolution computed tomography. Chest radiography might be inconclusive as in early stages it might be normal or nonspecific with features such as focal pneumonitis, scattered irregular opacities, linear or plate- like atelectasis. ${ }^{17}$ In the later stages chest radiography might be more specific for bronchiectasis with findings like specifically dilated and thickened airways that appear as ring-like shadows (of airways that are seen on end) or tram lines (in the case of airways that are perpendicular to the x-ray beam). 
Computed tomography findings of bronchiectasis includes 1) air-fluid levels in distended bronchi, 2) a linear array of cluster of cysts, 3) dilated peripheral bronchi, and 4) thickened bronchial walls. 5 In our case bronchiectasis was diagnosed by chest radiography and then confirmed by high resolution computed tomography. ${ }^{18}$

Many authors have tried to find out the risk factor for the development of bronchiectasis in HIV infections. It has been seen the bronchiectasis in HIV infected patients has been described in the presence of injection drug use, malnutrition, repeated opportunistic infection such as recurrent bacterial infections, pneumocystis jirovecii pneumonia, mycobacterium tuberculosis infection and LIP, severe viral lower respiratory tract infection. Bronchiectasis has been linked to the presence of HIV virus in lung tissue suggested by profound immunosuppresion with $\mathrm{CD} 4+$ $\mathrm{T}$ cells less than 100 cells $/ \mathrm{mm} 3$ or CDC immunology category 19,20,21,22.

Our child had repeated episodes of pneumonia, pulmonary tuberculosis and was malnourished which might have been the risk factors for development of bronchiectasis.

Bronchiectasis in children with HIV if not diagnosed early and managed aggressively can lead to irreversible destruction of bronchial wall and surrounding tissue leading to significant morbidity. This might decrease the benefit of the ART treatment on the long run. ${ }^{6}$

Treatment options for bronchiectasis are focused on symptom control, treatment of disease exacerbation and attempts to limit the rate of disease progression. In children with AIDS and bronchiectasis, high-protein, high-calorie diet should be encouraged to avoid weight loss associated with persistent infection. Regular exercise should be encouraged. There is reduced mucociliary clearance in bronchiectasis. Clearance of secretions with the help of postural drainage and chest percussion, along with assisted cough techniques, helps to improve the expectoration of mucus. Mechanical devices like oscillation jackets have not been efficacious. In our case, the patient was started on chest physiotherapy. ${ }^{5,23}$

Medical interventions include smoking cessation in adults, bronchodilators, oxygen supplementation, and management of exacerbations with antibiotics. HIV positive patients should also be offered Cotrimoxazole prophylaxis, Isoniazid preventive therapy, and could benefit from vaccination against Haemophilus influenzae and Streptococcus pneumonia. ${ }^{24}$ Very few data are available to evaluate the usefulness of chronic or intermittent prophylactic antibiotic therapy and questions as to the correct antibiotic to be used, the route of administration, and the duration of therapy need to be answered. Our patient was started on prophylaxis with Azithromycin weekly. ${ }^{23}$

Use of aerosolized recombinant human DNase used in $\mathrm{CF}$ have been shown to increase exacerbation frequency and decline in FEV1 among patients with non- CF bronchiectasis. $^{5}$

Given the direct cytotoxic effect of HIV infection, inhibition of HIV replication at the lung compartment through ART may further modulate the disease. It remains however, unclear if ART should be considered in all HIV infected patients with proven bronchiectasis, independent of CD4 count and clinical staging. ${ }^{25}$

Pulmonary resection may be an option for localized bronchiectasis and hemoptysis, but most physicians consider a trial of conservative treatment because lung tissue can appear bronchiectatic after infection for several weeks (pseudobronchiectasis) and surgery, if any, should be deferred until the status of the remaining lung tissue is well defined and may be more reasonable in patients with localized bronchiectasis or with ongoing symptoms affecting normal living. ${ }^{23}$

The occurrence of newly formed cystic lesions or bronchiectasis despite treatment may be risk factors for the development of pneumothorax or pneumomediastinum with subcutaneous emphysema in HIV infected patients. ${ }^{26}$

\section{Conclusion}

A high (15 to $42 \%)$ prevalence of bronchiectasis among people living with HIV has been reported. Given the high incidence of pulmonary infections and malnutrition, HIV positive persons in resource poor settings are at an even higher risk of developing pulmonary cystic disease. Bronchiectasis might become an increasingly important HIV associated co-morbidity. Due to the irreversible and progressive nature of the chronic lung disease, these pulmonary complications could compromise the benefits so a high index of suspicion should be kept for early diagnosis and treatment.

Conflict of interest: The authors declare that they have no conflict of interests.

\section{References}

1. Rosen J. Mark. Pulmonary complications of HIV infection. Respirology 2008; 13: 181-190

2. George R, Andronikou S, Theron S, Plesis J, Hayes M, Goussard P, Mapukata A, Gie R. Pulmonay infections in HIV-positive children. Pediatric Radiology 2009; 39(6):545- 554 
3. Rubinstein A, Morecki R, Silverman B, et al. Pulmonary disease in children with acquired immunodeficiency syndrome and AIDS-related complex. J Pediatr 1986; 108(4):498-503

4. Graham SM, Coulter JBS, Gilks CF. Pulmonary disease in HIV- infected African children. Int J Tuberc Lung Dis 2001; 5(1):12-23

5. Kenneth W. Tsang, Bilton D. Clinical challenges in managing bronchiectasis. Respirology. 2009; 14, 637650 .

6. Nicotra MB. Bronchiectasis. Semin Respir Infect 1994; $9(1): 31-40$

7. Banjar H. Childhood bronchiectasis: A review. Bahrain Medical Bulletin, 2006; 28(2)

8. Nikolaizik WH, Warner JO. Aetiology of chronic suppurative lung disease. Arch Dis Child 1994; $70(2): 141-42$

9. Eastham KM, Fall AJ, Mitchell L et al. The need to redefine non- cystic fibrosis bronchiectasis in childhood. Thorax 2004; 59(4): 324- 327.

10. Bard M, Couderc LJ, Saimot AG, Scherrer A, Frachon I, Seigneur F. Accelerated obstructive pulmonary disease in HIV infected patients with bronchiectasis. Eur Respir J 1998; 11(3): 771-775.

11.McGuinness G, Naidich DP, Garay S, et al. AIDS associated bronchiectasis: CT features. J Comput Assisted Tomogr 1993; 17(2): 260-266.

12.Moskovic E, Miller R, Pearson M. High resolution computed tomography of Pneumocystis carinii pneumonia in AIDS. Clin Radiol 1990; 42(4): 239-243.

13. Holmes AH, Trotman-Dickensen B, Edwards H, Peto TEA, Luzzi GA. Bronchiectasis in HIV Disease. Q J Med 1992; 307(2-3):875-82.

14. Holmes AH, Pelton S, Steinbach S, et al. HIV-related bronchiectasis (letter). Thorax 1995; 50(11): 1227.

15.Prasad R, Garg R, Sanjay. Bronchiectasis as a cause of unexplained breathlessness in HIV infected patient. The Internet Journal of Pulmonary Medicine 2007; 8(2)

16.Diaz PT, King MA, Pacht ER, Wewers MD, Gadek JE, Nagaraja HN, Drake J, Clanton TL: Increased susceptibility to pulmonary emphysema among HIV-seropositive smokers. Ann Intern Med 2000; 132(5):369-372

17.Barker AF: Bronchiectasis. N Engl J Med 2002; 346(18):1383-1393.
18. Callens SFJ, Kitetele F, Lelo P, Shabani N, Lusiama J, Wemakoy O, Colebunders R, Behets F, Rie AV. Pulmonary cystic disease in HIV positive individuals in the democratic republic of Congo: three case reports; Journal of Medical Case Reports 2007; 1(101):22

19. Van der Valk P, Monninkhof E, van der Palen J, Zielhuis G, van Herwaarden C: Management of stable COPD. Patient Educ Couns 2004; 52(3):225-229.

20. Sheikh S, Madiraju K, Steiner P, Rao M. Bronchiectasis in Pediatric AIDS. Chest 1997; 112(5): 1202-1207

21.Heather J. Zar. Chronic Lung disease in HIV infected children. Pediatric Pulmonology 2008; 43: 1-10.

22.Berman MD, Mafut D, Djokic B, Scott G, Mitchell C. Risk factors for the development of Bronchiectasis in HIV- infected children. Pediatric pulmonology 2007; 42: $871-875$

23.Lewiston NJ. Bronchiectasis. In: Hillman BC, ed. Pediatric respiratory disease: diagnosis and treatment. Philadelphia: WB Saunders 1993; 222-29

24.Keller DW, Breiman RF: Preventing bacterial respiratory tract infections among persons infected with human immunodeficiency virus. Clin Infect Dis 1995; 21 (Suppl 1):S77-83

25. Yearsley MM, Diaz PT, Knoell D, Nuovo GJ: Correlation of HIV-1 detection and histology in AIDS-associated emphysema. Diagn Mol Pathol 2005; 14(1):48-52

26. Cho JY, Kim DM, Ewon YE, Yoon SH, Lee SI. Newly formed cystic lesions for the development of pneumomediastinum in Pneumocystis jirovecii pneumonia. BMC Infectious Diseases 2009; 9(171):18 